\title{
Localization of SUCLA2 and SUCLG2 subunits of succinyl CoA ligase within the cerebral cortex suggests the absence of matrix substrate-level phosphorylation in glial cells of the human brain
}

\author{
Arpád Dobolyi • Attila G. Bagó • Aniko Gál • \\ Mária J. Molnár • Miklós Palkovits • Vera Adam-Vizi • \\ Christos Chinopoulos
}

Received: 11 July 2014 / Accepted: 21 September 2014

(C) Springer Science+Business Media New York 2014

\begin{abstract}
We have recently shown that the ATP-forming SUCLA2 subunit of succinyl-CoA ligase, an enzyme of the citric acid cycle, is exclusively expressed in neurons of the human cerebral cortex; GFAP- and S100-positive astroglial cells did not exhibit immunohistoreactivity or in situ hybridization reactivity for either SUCLA2 or the GTP-forming SUCLG2. However, Western blotting of post mortem samples revealed a minor SUCLG2 immunoreactivity. In the present
\end{abstract}

A. Dobolyi

MTA-ELTE NAP Laboratory of Molecular and Systems

Neurobiology, Institute of Biology, Budapest, Hungary

A. Dobolyi $\cdot$ M. Palkovits

Department of Anatomy, Histology and Embryology, Semmelweis

University, Budapest, Hungary

A. G. Bagó

National Institute of Clinical Neurosciences, Budapest, Hungary

A. Gál • M. J. Molnár

Institute of Genomic Medicine and Rare Disorders, Semmelweis

University, Budapest, Hungary

M. Palkovits

Human Brain Tissue Bank and Laboratory, Semmelweis University, Budapest, Hungary

V. Adam-Vizi

Department of Medical Biochemistry, Semmelweis University,

Hungarian Academy of Sciences-SE Laboratory for

Neurobiochemistry, Budapest, Hungary

C. Chinopoulos $(\bowtie)$

Department of Medical Biochemistry, Semmelweis University,

Budapest, Hungary

e-mail: chinopoulos.christos@eok.sote.hu

C. Chinopoulos

MTA-SE Lendület Neurobiochemistry Research Group, Budapest, Hungary work we sought to identify the cell type(s) harboring SUCLG2 in paraformaldehyde-fixed, free-floating surgical human cortical tissue samples. Specificity of SUCLG2 antiserum was supported by co-localization with mitotracker orange staining of paraformaldehyde-fixed human fibroblast cultures, delineating the mitochondrial network. In human cortical tissue samples, microglia and oligodendroglia were identified by antibodies directed against Iba1 and myelin basic protein, respectively. Double immunofluorescence for SUCLG2 and Iba1 or myelin basic protein exhibited no costaining; instead, SUCLG2 appeared to outline the cerebral microvasculature. In accordance to our previous work there was no co-localization of SUCLA2 immunoreactivity with either Iba1 or myelin basic protein. We conclude that SUCLG2 exist only in cells forming the vasculature or its contents in the human brain. The absence of SUCLA2 and SUCLG2 in human glia is in compliance with the presence of alternative pathways occurring in these cells, namely the GABA shunt and ketone body metabolism which do not require succinyl CoA ligase activity, and glutamate dehydrogenase 1, an enzyme exhibiting exquisite sensitivity to inhibition by GTP.

Keywords Krebs cycle $\cdot$ Citric acid cycle Substrate-level phosphorylation - Succinyl CoA ligase $\cdot$ Microglia .

Oligodendrocytes

\section{Introduction}

Succinyl CoA ligase (SUCL) is a heterodimeric enzyme, composed of an invariant $\alpha$ subunit encoded by SUCLG1 and a substrate-specific $\beta$ subunit, encoded by either SUCLA2 or SUCLG2. This dimer combination results in either an ATP-forming (EC 6.2.1.5) or a GTP-forming 
SUCL (EC 6.2.1.4). The enzyme catalyzes the conversion of succinyl-CoA and ADP (or GDP) to CoASH, succinate and ATP (or GTP) in the mitochondrial matrix, as part of the citric acid cycle (Johnson et al. 1998a). $\Delta G$ for this reaction is $0.07 \mathrm{~kJ} / \mathrm{mol}$ and therefore it is reversible (Li et al. 2013).

In frequently used laboratory animals such as rodents, SUCLA2 and SUCLG2 exhibit quantitative differences in a tissue-specific expression, as opposed to humans where differential expression in some tissues appears to be qualitative (Johnson et al. 1998b; Lambeth et al. 2004). Characteristically, in the human brain SUCLA2 is found only in the neurons, while SUCLG2 is not found either in neurons or in glial fibrillary acidic protein (GFAP)- and S100-positive astroglial cells (Dobolyi et al. 2013).

Mindful of the weak immunoreactivity for SUCLG2 in Western blotting experiments from post mortem human brain samples (Dobolyi et al. 2013), we sought to identify the cell type(s) harboring mitochondria with this protein. In our previous study, we identified neurons and astrocytes using the markers Nissl and GFAP/S100, respectively (Dobolyi et al. 2013). In the present work we extended our investigation by including the microglial marker ionized calcium-binding adapter molecule 1 (Iba1) and oligodendroglial marker myelin basic protein.

\section{Materials and methods}

Cell cultures Fibroblast culture from skin biopsies from a control subject was prepared. Cells were grown on poly-Lornithine coated $25 \mathrm{~mm}$ round glass coverslips for 5-7 days, at a density of approximately $8 * 10^{5}$ cells/coverslip in RPMI1640 medium (GIBCO, Life technologies, Carlsbad, CA, USA) supplemented with $10 \%$ fetal bovine serum and $2 \mathrm{mM}$ glutamine and kept at $37{ }^{\circ} \mathrm{C}$ in $5 \% \mathrm{CO}_{2}$. The medium was also supplemented with penicillin, streptomycin and amphotericin (item A5955, Sigma-Aldrich St. Louis, MO, USA).

Immunocytochemistry of cell cultures Fibroblasts cultures were first treated with $1 \mu \mathrm{M}$ Mitotracker Orange (MTO) for $5 \mathrm{~min}$ in their culture media, at $37^{\circ} \mathrm{C}$ in $5 \% \mathrm{CO}_{2}$. Subsequent immunocytochemistry of the cultures was performed by fixing the cells with $4 \%$ paraformaldehyde in phosphatebuffered saline (PBS) for $20 \mathrm{~min}$, followed by permeabilization by $0.1 \%$ TX-100 (in PBS) for $10 \mathrm{~min}$ and several washing steps in between with PBS at room temperature. Cultures were treated with $10 \%$ donkey serum overnight at $4{ }^{\circ} \mathrm{C}$ followed by bathing in $1 \%$ donkey serum and $0.175 \mu \mathrm{g} / \mathrm{ml}$ anti-SUCLG2 (Atlas Antibodies AB, AlbaNova University Center, Stockholm, Sweden, Cat. No. HPA046705) for $1 \mathrm{~h}$, at room temperature. This antibody was raised by immunizing rabbits with a recombinant fragment corresponding to the amino acid region 363-432 of human SUCLG2 (NP 003839). Therefore, it should not be able to distinguish among transcript variants (Dobolyi et al. 2013). Cells were subsequently decorated by using the appropriate Alexa 488-linked secondary antibody $(1: 5,000$, donkey antirabbit, Jackson Immunochemicals Europe Ltd, Cambridgeshire, UK) in the presence of $1 \%$ donkey serum.

Human brains Human brain samples were collected in accordance with the Ethical Rules for Using Human Tissues for Medical Research in Hungary (HM 34/1999) and the Code of Ethics of the World Medical Association (Declaration of Helsinki). Surgical brain samples were obtained from tissue removed during brain surgeries at the National Institute of Clinical Neurosciences, Budapest, Hungary in the framework of the Human Brain Tissue Bank and Laboratory, Semmelweis University, Budapest. Prior written informed consent was obtained from the patients that included the request to conduct neurochemical analyses. The protocols including analyses of tissue samples were approved by institutional ethics committee of the Semmelweis University. The surgical patients underwent the removal of brain tumors. The medical history of the subjects was obtained from medical or hospital records, interviews with family members and relatives, as well as from pathological and neuropathological reports. All personal identifiers had been removed and samples were coded before the analyses of tissue. Immunolabeling was performed using frontal cortical sample of a 58 years old man and temporal cortical samples from 64 to 66 years old women.

Tissue collection for immunolabeling For immunocytochemistry, brains were cut into 5-10 mm thick coronal slices and immersion fixed in $4 \%$ paraformaldehyde in $0.1 \mathrm{M}$ PBS for 3-5 days. Subsequently, the blocks were transferred to PBS containing $0.1 \%$ sodium azide for 2 days to remove excess paraformaldehyde. Then the blocks were placed in PBS containing $20 \%$ sucrose for 2 days for cryoprotection, after which the blocks were frozen and cut into $50 \mu \mathrm{m}$ thick serial coronal sections on a sliding microtome. Sections were collected in PBS containing $0.1 \%$ sodium azide and stored at $4{ }^{\circ} \mathrm{C}$ until further processing.

Double labeling of SUCLA2 and SUCLG2 with glial markers in brains sections Every fifth free-floating brain section of human temporal and frontal cortical blocks was immunostained for SUCLA2, SUCLG2, and for glial markers. First, the glial markers were applied. Oligodendrocytes were labeled with a mouse anti-myelin basic protein antibody (1:100, Abcam, Cambridge, UK; cat. number: ab24567) while microglia were stained with goat anti-Ibal (1:500, Abcam; cat. number: ab107159). Thus, the sections were incubated in the primary antibodies for $24 \mathrm{~h}$ at room temperature, then in Alexa 
594 donkey anti-mouse or anti-goat secondary antibodies (1:500; Molecular Probes, Eugene, OR) for $2 \mathrm{~h}$ and washed. Subsequently, rabbit anti-SUCLA2 (Proteintech Europe Ltd, Manchester, UK, Cat. No. \# 12627-1-AP) and antiSUCLG2 antisera were applied using 1:1,000 dilution followed by incubation of the sections in biotinylated anti-rabbit secondary antibody (1:1,000 dilution; Vector Laboratories, Burlingame, CA) and then in avidinbiotin-peroxidase complex (1:500; Vector Laboratories) for $2 \mathrm{~h}$. Subsequently, the labeling was visualized by incubation in fluorescein isothiocyanate (FITC)-tyramide $(1: 8,000)$ and $\mathrm{H}_{2} \mathrm{O}_{2}$ in $100 \mathrm{mM}$ Trizma buffer $(\mathrm{pH} 8.0$ adjusted with $\mathrm{HCl}$ ) for 6 min. Finally, all sections were mounted on positively charged slides (Superfrost Plus, Fisher Scientific, Pittsburgh, PA) and coverslipped in antifade medium (Prolong Antifade Kit, Molecular Probes).

Image processing Fluorescent sections were evaluated using a Bio-Rad 2100 Rainbow Confocal System (Bio-Rad Laboratories, Inc, CA, USA). For the human brain specimens, pictures were taken at the frequencies of the two dyes and also in the far red range. This latter image was subtracted from both of the other two images in order to cancel-out significant autofluorescence of the sections which was detected in all channels. Subsequently, the contrast and sharpness of the images were adjusted using the "levels" and "sharpness" commands in Adobe Photoshop CS 8.0. Full resolution was maintained until the photomicrographs were finally cropped at which point the images were adjusted to a resolution of 300 dpi.

\section{Results}

The specificity of SUCLG2 immunoreactivity in human fibroblast cultures

We have used a new antibody directed against the human SUCLG2 protein that does not distinguish among various isoforms (see "Materials and Methods") as in (Dobolyi et al. 2013). The specificity of SUCLG2 immunoreactivity was validated using human fibroblast cells, see Fig. 1. The mitochondrial network was selectively stained by loading cells with Mitotracker Orange (MTO, $1 \mu \mathrm{M})$ prior to fixation (panel a). Fibroblasts were subsequently labeled with the procedure of SUCLG2 immunostaining (panel b), and there was a co-localization with the decoration obtained by MTO (panel c). Note that only a minority of the mitochondrial network was stained by the SUCLG2 antiserum, as opposed to SUCLA2 immunoreactivity in the same cells, in which the
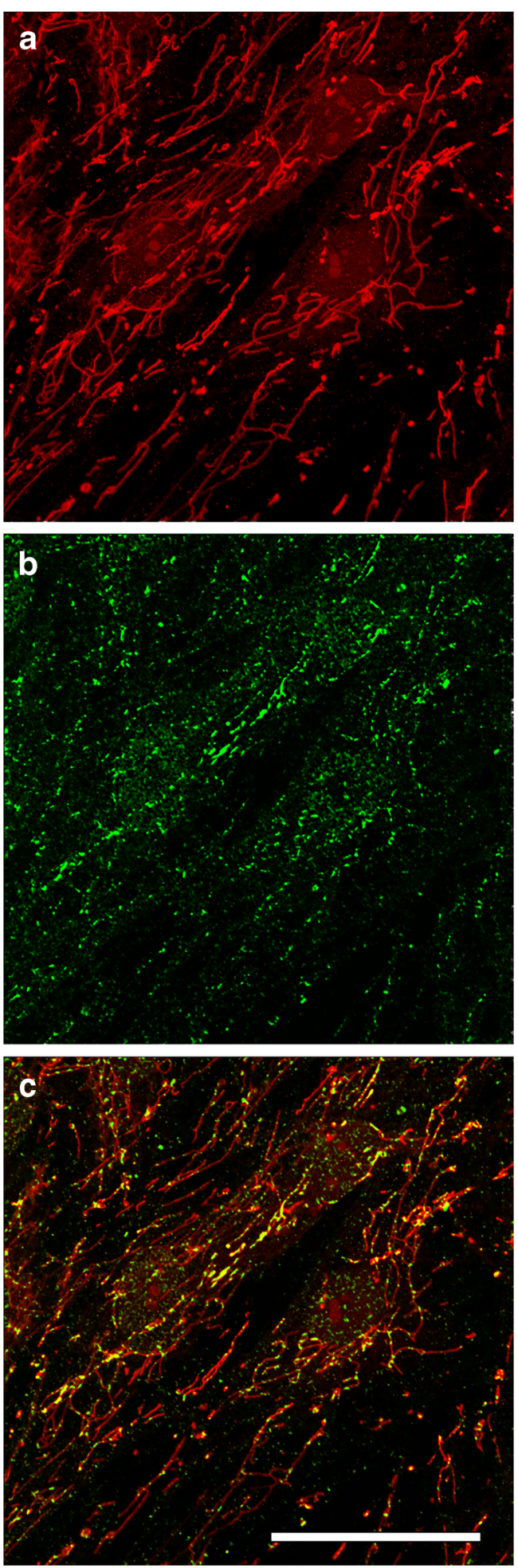

Fig. 1 Co-labeling of human fibroblasts (SUCLG2) with Mitotracker Orange (MTO). a MTO labeling outlining the mitochondrial network in normal human fibroblasts. b SUCLG2 immunoreactivity in normal human fibroblasts. Intracellular structures resembling mitochondria are intensely labeled $\mathbf{c}$ Merged image of SUCLG2 immunodecoration with MTO labeling. Scale bar $=13 \mu \mathrm{m}$

majority of the mitochondrial network was stained (Dobolyi et al. 2013). 


\section{SUCLA2 and SUCLG2 labeling in the cerebral cortex}

SUCLA2 immunoreactivity appeared in cells located throughout the cerebral cortex (Fig. 2a). The cellular distribution of SUCLA2 labeling suggested that SUCLA2 immunoreactivity is restricted to mitochondria (see Fig. 3a), exactly as described by us previously (Dobolyi et al. 2013). Furthermore, the distribution of SUCLA2-immunoreactive cells corresponded to that of neurons in accordance with the previously described neuronal expression of this protein. In these sections, the walls of large blood vessels were also labeled with SUCLA2, see upper left corner of figure panel 2a. The distribution of SUCLA2 immunoreactivity differed markedly from that of myelin basic protein as the latter one was striking in the white matter and labeled radially arranged structures in accordance with the established localization of myelinated fibers within the cortex (Fig. 2b). Indeed, double labeling confirmed the absence of co-localization between SUCLA2 and myelin basic protein (Figs. 2c, 3b). The distribution of Ibal-labeled microglial cells was relatively even within the examined brain section corresponding to the role of this cell type in immune defense (Fig. 2e). Microglial cells had small somata and several processes (Fig. 3a). Thus, the morphology of SUCLA2 and Iba1-labeled cells was different. Consistent with this, no co-localization of these markers were found in any cortical regions examined (Fig. 3a).
Fig. 2 Immunocytochemical identification of succinyl CoA ligase subunits, oligodendroglia, and microglia in the human cerebral cortex. The distribution of SUCLA2 (a), myelin basic protein (b), SUCLG2 (d) and Iba1 (e) immunoreactivities are shown in low magnification. c Double labeling (panels a and $\mathbf{b}$ combined) show somewhat overlapping but still fundamentally different distributions of SUCLA2 and myelin basic protein. f Double labeling (panels $\mathbf{d}$ and $\mathbf{e}$ combined) demonstrate the distinct distributions of SUCLG2 and Ibal in the cerebral cortex. Scale bar $=500 \mu \mathrm{m}$
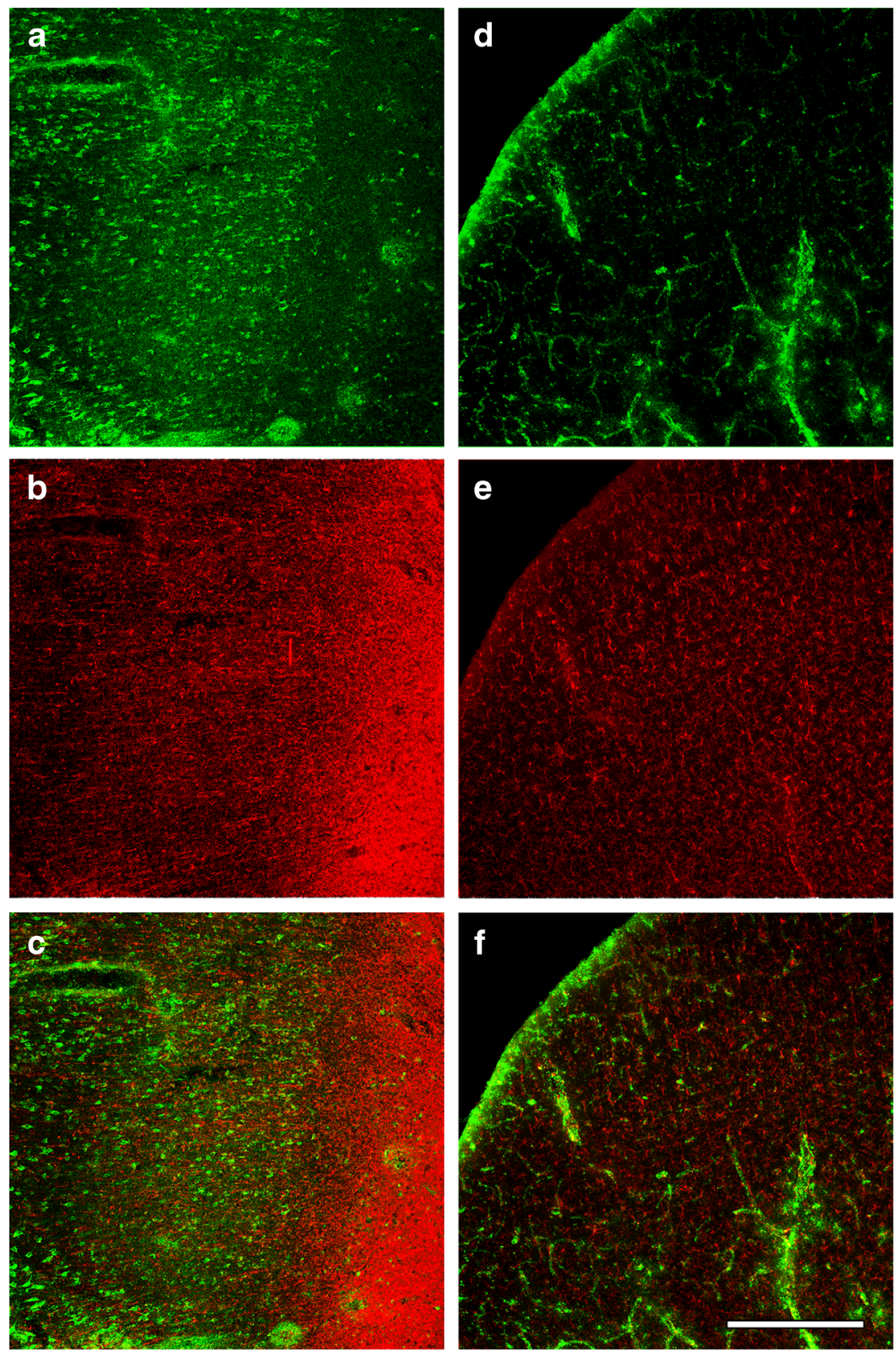
Fig. 3 High magnification confocal images demonstrate that SUCLA2 immunoreactivity (green in $\mathbf{a}$ and $\mathbf{b}$ ) is present in neurons but not in Iba1-positive microglia cells (red in a) and in oligodendroglial cells labelled with basic myelin protein (red in b). In turn, SUCLG2

immunoreactivity (green in $\mathbf{c}$ and d) does not co-localize with Iba1 (red microglia cells in c) and cells labelled with basic myelin protein (red oligodendroglial cells in d).

The arrowheads in $\mathbf{d}$ point to red blood cells within vessels labelled non-specifically due to their internal peroxidase content. Scale bar $=20 \mu \mathrm{m}$
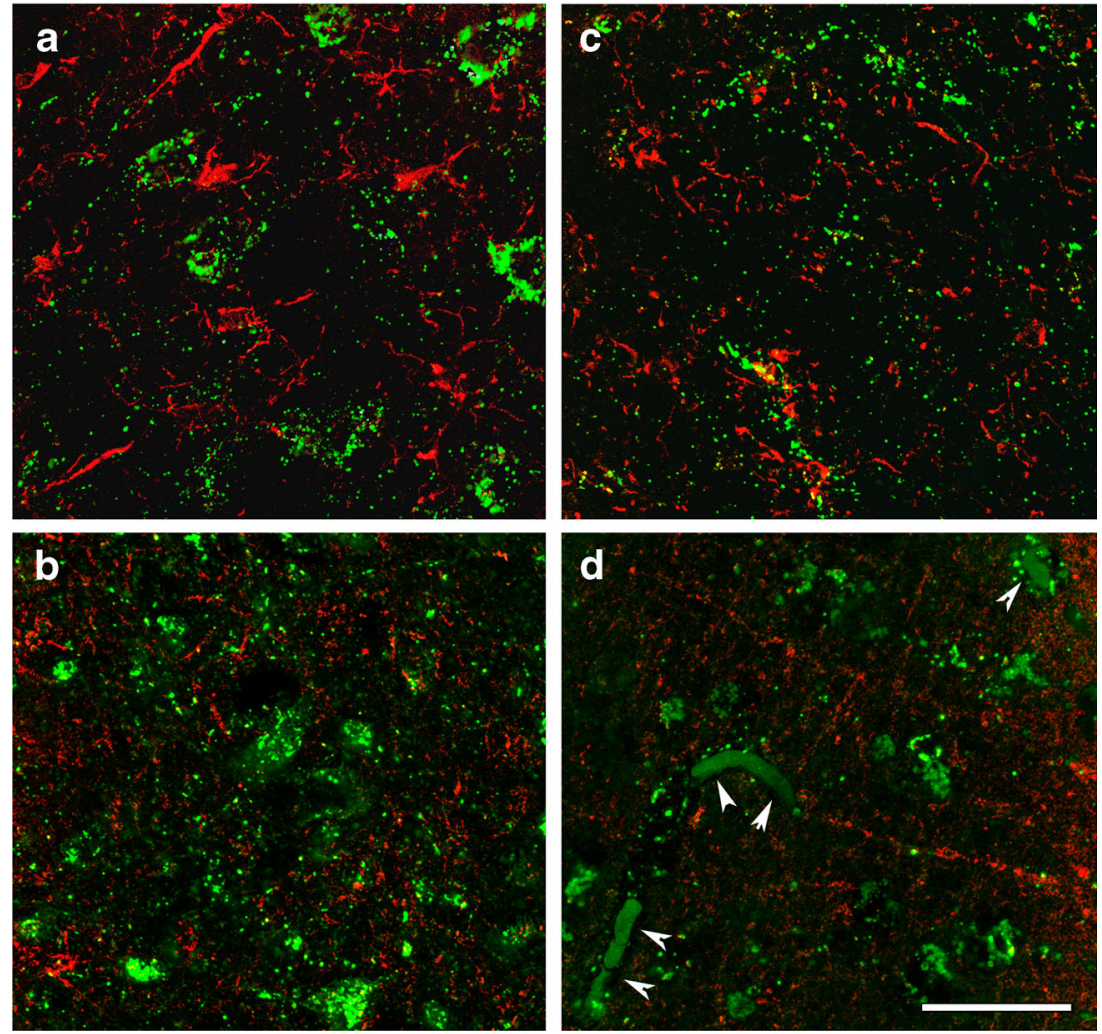

SUCLG2 immunoreactivity was found in all layers of the human cerebral cortex. Its distribution was, however, characteristic of blood vessels suggesting endothelial and/or pericytic localization. However, we must note that perivascular staining due to the presence of SUCLG2 in astrocyte end-feet is a viable possibility. The distribution of SUCLG2 immunoreactivity was clearly different from that of myelin basic protein (Fig. 2b) and Iba1-positive microglia (Fig. 2e), which was also confirmed by double labeling (Fig. 2f). Analysis of high magnification images revealed that SUCLG2 immunoreactivity present in structures resembling mitochondria appeared in capillary-forming cells. This is best depicted in the lower left corner (two white arrowheads) and upper right corner (single white arrowhead) of figure panel $3 \mathrm{~d}$; note the SUCLG2 decoration pattern forming a tubular structure around the red blood cells (stained green because of the immunohistochemical procedure, detailed in "Materials and Methods") in a longitudinal (lower left corner) and crosssection (upper right corner) mode delineating a capillary. No co-localization was found between SUCLG2 and the microglia marker Iba1 (Fig. 3c) and oligodendroglial marker myelin basic protein (Fig. 3d). On the other hand, SUCLG2 expression has been demonstrated in microglial cells of post-mortem brains of Alzheimer's disease patients and age-matched controls (Ramirez et al. 2014).

In humans, SUCLG2 exhibits transcript variants. However, the possibility that the human glial transcript variant of
SUCLG2 is not recognized by existing antibodies has been ruled out in (Dobolyi et al. 2013): all transcripts are identical in the region 1-396, except in position 220 (Dobolyi et al. 2013). The antibody used in (Dobolyi et al. 2013) to identify SUCLG2 was raised by immunizing rabbits with a recombinant fragment corresponding to a region within the $\mathrm{N}$ terminal amino acids 1-204 of human SUCLG2 which is common among all transcript variants. Furthermore, in (Dobolyi et al. 2013), PCR and in situ hybridization for all known variants have been performed for human brain material; SUCLG2 mRNA was deduced to be very scarce in the human brain, while the same probes tested robustly positive in human fibroblasts.

\section{Discussion}

The present article is a follow-up on the lead provided by our previous work showing that SUCLG2 is very weakly expressed in the human brain, and it is not found in either neurons or astrocytes (Dobolyi et al. 2013). Here, the most important finding is that SUCLG2 expression was further confirmed to be absent from microglia and oligodendroglia, but it was detected in cells forming the microvasculature, most likely endothelial cells and/or pericytes. 
An additional finding is that SUCLG2 staining in human fibroblasts covered only a small fraction of the mitochondrial network. On the other hand, SUCLA2 staining covered the majority of mitochondria in the same cells (Dobolyi et al. 2013). It would be interesting to see a triple-colocalization study of SUCLG2, SUCLA2 and mitotracker orange in the same cell; a non-overlapping SUCLA2-SUCLG2 staining would signify the presence of two different mitochondrial populations regarding succinyl CoA ligase activity, one representing the ATP-forming population, and the other the GTP-forming tier. This would not be too surprising; ATP is associated with catabolic pathways, while GTP with anabolism (Pall 1985). GTP is very valuable in the mitochondrial matrix, as it cannot be transported through the inner mitochondrial membrane of higher organisms (Pfaff et al. 1965), hence it also regenerated through salvage reactions. Currently, such a co-staining is not possible or at least technically very challenging because both available antibodies directed against SUCLA2 and SUCLG2 are raised in rabbits. We are in the process of generating a SUCLG2 antibody raised in goats directed against the same epitope as the present anti-rabbit antibody, for the purpose of simultaneous SUCLA2 and SUCLG2 co-staining.

The characterization of cell-specific SUCLA2 and SUCLG2 expression is significant because the simultaneous absence of both proteins in any cell imply a missing step in the citric acid cycle as we know it. Apparently, this seems to be the case for astrocytes, microglia, and oligodendrocytes in the human brain. The question arises, how can these cells perform normal metabolic functions, in the absence of an intact citric acid cycle?

To address this, we must first elaborate on the relevant biochemical pathways. As it is mentioned in the "Introduction", succinyl CoA ligase is a reversible enzyme; when it proceeds towards generation of succinyl-CoA, this product may only follow heme- or ketone body metabolism (Labbe et al. 1965; Ottaway et al. 1981; Dringen et al. 2007; Lopes-Cardozo et al. 1986) because it cannot be processed further by the irreversible $\alpha$-ketoglutarate dehydrogenase complex (Chinopoulos 2013). When succinyl CoA ligase operates in the opposite direction towards ATP or GTP formation (a process termed 'substratelevel phosphorylation'), it yields succinate that can be either further processed by succinate dehydrogenase, and advance in the citric acid cycle, or act as a metabolic signal in inflammation (Mills and O'Neill 2014).

In the human brain, there are at least two biochemical pathways that can mediate a by-pass of succinyl CoA ligase: i) the 'GABA shunt', and ii) ketone metabolism.

The 'GABA shunt' (Fig. 4) is a pathway commencing from glutamate (which could arise from $\alpha$-ketoglutarate through glutamate dehydrogenase) being converted to GABA by the cytosolic glutamate decarboxylase (4.1.1.15), encoded by either GAD65 or GAD67 (Soghomonian and Martin 1998). GABA may also arise by metabolism of putrescine

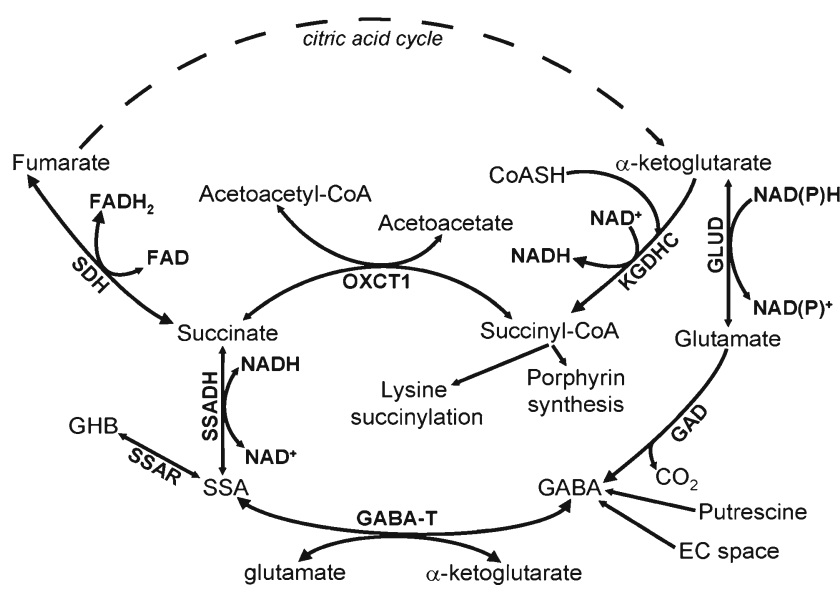

Fig. 4 Illustration of the pathways bypassing succinyl CoA ligase in human glia. $K G D H C$ ketoglutarate dehydrogenase complex, GLUD glutamate dehydrogenase, $G A D$ glutamate decarboxylase, GABA $\gamma$ aminobutyric acid, EC space extracellular space, GABA-T GABA transaminase, SSA succinate semialdehyde, SSADH succinate semialdehyde dehydrogenase, SSAR succinate semialdehyde reductase, GHB $\gamma$ hydroxybutyric acid, $S D H$ succinate dehydrogenase, OXCT1 succinylCoA:3-ketoacid-coenzyme A transferase 1

(Sequerra et al. 2007; Jakoby and Fredericks 1959; De Mello et al. 1976; Seiler et al. 1973), or enter the cytoplasm from the extracellular space through transporters in the plasma membrane. GABA transaminates with $\alpha$-ketoglutarate to form glutamate and succinate semialdehyde by the mitochondrial GABA transaminase (GABA-T, EC 2.6.1.19). Succinate semialdehyde will get dehydrogenated by the $\mathrm{NAD}^{+}$-dependent succinate semialdehyde dehydrogenase (EC 1.2.1.24), encoded by aldehyde dehydrogenase 5 family, member A1 (ALDH5Al) to yield succinate, and thus enter the citric acid cycle. Succinate semialdehyde can also be converted to gamma-hydroxybutyrate by succinic semialdehyde reductase, encoded by AKR7A2 (Picklo et al. 2001), an enzyme that is primarily localized to astrocytes and microglia of the human cerebral cortex, hippocampus and midbrain (Picklo et al. 2001; Ris and von Wartburg 1973; Hoffman et al. 1980).

The enzymes required for a complete 'GABA shunt' are present in glial cells in the adult human brain; astrocytes express glutamate dehydrogenase (both isoforms 1 and 2, see below) (Spanaki et al. 2014), GAD67 (but not GAD65), GABA-T as well as the $\mathrm{GABA}_{\mathrm{A}}$ and $\mathrm{GABA}_{\mathrm{B}}$ receptors (Lee et al. 2011a; Schwab et al. 2013) reviewed in (Velez-Fort et al. 2012). The intensity of immunostaining for GAD67 and GABA-T in these cells is comparable or greater to that observed for known inhibitory neurons. The GABA transporter GAT-3 is exclusively expressed in astrocytes (Minelli et al. 1996); GAT-1 and -2 are found in astrocytic processes and other neuronal- and nonneuronal elements (Conti et al. 1998, 1999). As a result of the concerted action of these enzymes and transporters, cultured human astrocytes maintain an intracellular GABA level of $2.32 \mathrm{mM}$ (Lee et al. 2011b). On the same line, cultured human microglia express the mRNA and protein for GABA-T, in 
addition to $\mathrm{GABA}_{\mathrm{A}}$ and $\mathrm{GABA}_{\mathrm{B}}$ receptors (Lee et al. 2011a). The $\mathrm{NAD}^{+}$-dependent succinate semialdehyde dehydrogenase encoded by $A L D H 5 A 1$ is expressed in both neurons and glial cells in the adult human brain (http://www.proteinatlas.org/ ENSG00000112294/tissue).

Regarding ketone bodies metabolism, it is well established that 3-hydroxybutyrate and acetoacetate can replace glucose as the major source of brain energy in situations such as starvation (Robinson and Williamson 1980). Ketone bodies are not only a product of the liver, but also of astrocytes: astrocytes produce ketone bodies from fatty acids (Auestad et al. 1991), and the branched-chain amino acids valine, isoleucine and leucine (Murin et al. 2009a, b; Bixel and Hamprecht 1995). A critical enzyme in ketone body metabolism is the mitochondrial succinyl-CoA:3-ketoacid-coenzyme A transferase 1 (EC 2.8.3.5) (Hersh and Jencks 1967), encoded by OXCT1. This enzyme catalyzes the reversible transfer of $\mathrm{CoA}$ from succinyl $\mathrm{CoA}$ to acetoacetate yielding acetoacetyl $\mathrm{CoA}$ and succinate (Fig. 4). OXCT1 is expressed in both neurons and glial cells in the human brain (http://www.proteinatlas.org/ENSG00000083720/tissue). From the reaction catalyzed by succinyl-CoA:3-ketoacidcoenzyme A transferase 1, it is apparent that there can be interconversion of succinate to succinyl $\mathrm{CoA}$ and vice versa, obviating the need for succinyl CoA ligase.

From the above, it is evident that human glia can accommodate for the lack of succinyl CoA ligase with the 'GABA shunt' and/or ketone metabolism, and thus maintain production of reducing equivalents by the citric acid cycle. However, it would be erroneous to consider that the 'GABA shunt' and ketone bodies metabolic pathways evolved in these cells in response to the lack of succinyl CoA ligase. Glia benefit from shuttling succinyl CoA towards GABA formation and ketone body metabolism: i) GABA is an effective immunomodulatory molecule (Jin et al. 2013); in human astrocytes and microglia, GABA suppresses the reactive response of the inflammatory stimulants lipopolysaccharide and interferon- $\gamma$ by inhibiting the induction of inflammatory pathways mediated by NFKB and P38 MAP kinase (Lee et al. 2011a); ii) Ketone bodies produced by the astrocytes may promote neuroprotection by supplying them to the neurons (Guzman and Blazquez 2001).

Alternatively, in the absence of succinyl-CoA ligase, succinylCoA emerging from the $\alpha$-ketoglutarate dehydrogenase complex in glial mitochondria could serve as cofactor for lysine succinylation, a wide-spread post-translational modification (Zhang et al. 2011), or advance towards porphyrin synthesis.

Furthermore, the absence of a GTP-forming succinyl CoA ligase (and likewise, of an ATP-forming succinyl CoA ligase, since ATP can transphosphorylate to GTP by the concerted action of SUCL with a mitochondrial isoform of a nucleotide diphosphate kinase known as nm23-H4 (Kadrmas et al. 1991; Kowluru et al. 2002)) is compatible with the high level of expression of glutamate dehydrogenase (GLUD) in human glia (Plaitakis and Zaganas 2001): In the human brain, GLUD is encoded by either GLUD1 or GLUD2 (Plaitakis and Zaganas 2001). GLUD1 is potently inhibited by GTP $\left(\mathrm{IC}_{50}=0.20 \mu \mathrm{M}\right)$, (Plaitakis and Zaganas 2001). GLUD2 is refractory to GTP, but it is strongly activated by ADP and leucine (Spanaki et al. 2012). However, GLUD2 expression is much lower than GLUD1 (Zaganas et al. 2012). Both GLUD isoforms are mostly expressed in the astrocytes; oligodendroglia or myelinated fibers do not express GLUD2 (Spanaki et al. 2010). To the best of out knowledge, there are no published data on GLUD2 expression in microglia.

Although the above considerations in conjunction with our results point to the direction of a truncated citric acid cycle in human glia, experimental evidence that support or refute this concept in glia within the adult human brain are as of yet, lacking; substrate-specific metabolism of in situ astrocytic mitochondria deduced by isotopomeric analysis of ${ }^{13} \mathrm{C}$ distributed among various metabolites has been extensively addressed but only in cell-specific cultures from rodent brain originating from fetal materials that mature on entirely artificial conditions (Westergaard et al. 1994; Schousboe et al. 1997; Hassel 2000; McKenna and Sonnewald 2005). Therefore, extrapolations from such experiments on what could be actually happening in glia within the adult human brain -as suggested in Fig. 4 - must be exercised with caution.

A potential 'downside' for glial cells lacking the capacity for nucleotide-forming SUCL (substrate-level phosphorylation) is that their mitochondria are likely to engage in cytosolic ATP consumption (Chinopoulos et al. 2010; Kiss et al. 2013, 2014; Chinopoulos 2011a), during natural fluctuations of their membrane potentials (Gerencser et al. 2012) should they become sufficiently depolarized. By the same token, glial mitochondria should also be vulnerable in terms of relying on -in house- ATP reserves during energy crisis, such as during brain ischemia (Chinopoulos 2011b; Chinopoulos and Adam-Vizi 2010).

Acknowledgments Thanks are expressed to Katalin Zölde for excellent technical assistance.

Funding This work was supported by the KTIA_NAP_B 13-2-20140004 and the Országos Tudományos Kutatási Alapprogram (OTKA) K 100319 grants to A.D., OTKA 81983 and the Hungarian Academy of Sciences grants 02001 to V. A.-V, TÁMOP 4.2.1./B-09/1/KMR and NAP-A grants to M.J.M. and A.G., OTKA grants NNF 78905, NNF2 85658, K 100918, and the MTA-SE Lendület Neurobiochemistry Research Division grant 95003 to C.C.

Conflict of interest The authors declare no conflict of interest.

\section{References}

Auestad N, Korsak RA, Morrow JW, Edmond J (1991) Fatty acid oxidation and ketogenesis by astrocytes in primary culture. $\mathrm{J}$ Neurochem 56(4):1376-1386 
Bixel MG, Hamprecht B (1995) Generation of ketone bodies from leucine by cultured astroglial cells. J Neurochem 65(6):2450-2461

Chinopoulos C (2011a) The "B Space" of mitochondrial phosphorylation. J Neurosci Res 89(12):1897-1904. doi:10.1002/jnr.22659

Chinopoulos C (2011b) Mitochondrial consumption of cytosolic ATP: not so fast. FEBS Lett 585(9):1255-1259. doi:10.1016/j.febslet. 2011.04.004

Chinopoulos C (2013) Which way does the citric acid cycle turn during hypoxia? The critical role of alpha-ketoglutarate dehydrogenase complex. J Neurosci Res 91(8):1030-1043. doi:10.1002/jnr.23196

Chinopoulos C, Adam-Vizi V (2010) Mitochondria as ATP consumers in cellular pathology. Biochim Biophys Acta 1802(1):221-227. doi: 10.1016/j.bbadis.2009.08.008

Chinopoulos C, Gerencser AA, Mandi M, Mathe K, Torocsik B, Doczi J et al (2010) Forward operation of adenine nucleotide translocase during F0F1-ATPase reversal: critical role of matrix substrate-level phosphorylation. FASEB J 24(7):2405-2416. doi:10.1096/fj.09149898

Conti F, Melone M, De BS, Minelli A, Brecha NC, Ducati A (1998) Neuronal and glial localization of GAT-1, a high-affinity gammaaminobutyric acid plasma membrane transporter, in human cerebral cortex: with a note on its distribution in monkey cortex. J Comp Neurol 396(1):51-63. doi:10.1002/(SICI)1096-9861(19980622) $396: 1<51::$ AID-CNE5 $>3.0$. CO; $2-\mathrm{H}$

Conti F, Zuccarello LV, Barbaresi P, Minelli A, Brecha NC, Melone M (1999) Neuronal, glial, and epithelial localization of gammaaminobutyric acid transporter 2, a high-affinity gammaaminobutyric acid plasma membrane transporter, in the cerebral cortex and neighboring structures. J Comp Neurol 409(3):482494. doi:10.1002/(SICI)1096-9861(19990705)409:3<482::AIDCNE11>3.0.CO;2-O

De Mello FG, Bachrach U, Nirenberg M (1976) Ornithine and glutamic acid decarboxylase activities in the developing chick retina. $\mathrm{J}$ Neurochem 27(4):847-851

Dobolyi A, Ostergaard E, Bago AG, Doczi T, Palkovits M, Gal A et al (2013) Exclusive neuronal expression of SUCLA2 in the human brain. Brain Struct Funct. doi:10.1007/s00429-013-0643-2

Dringen R, Bishop GM, Koeppe M, Dang TN, Robinson SR (2007) The pivotal role of astrocytes in the metabolism of iron in the brain. Neurochem Res 32(11):1884-1890. doi:10.1007/s11064-007-9375-0

Gerencser AA, Chinopoulos C, Birket MJ, Jastroch M, Vitelli C, Nicholls DG et al (2012) Quantitative measurement of mitochondrial membrane potential in cultured cells: calcium-induced de- and hyperpolarization of neuronal mitochondria. J Physiol 590(Pt 12):28452871. doi:10.1113/jphysiol.2012.228387

Guzman M, Blazquez C (2001) Is there an astrocyte-neuron ketone body shuttle? Trends Endocrinol Metab 12(4):169-173

Hassel B (2000) Carboxylation and anaplerosis in neurons and glia. Mol Neurobiol 22(1-3):21-40. doi:10.1385/MN:22:1-3:021

Hersh LB, Jencks WP (1967) Coenzyme A transferase: kinetics and exchange reactions. J Biol Chem 242(15):3468-3480

Hoffman PL, Wermuth B, von Wartburg JP (1980) Human brain aldehyde reductases: relationship to succinic semialdehyde reductase and aldose reductase. J Neurochem 35(2):354-366

Jakoby WB, Fredericks J (1959) Pyrrolidine and putrescine metabolism: gamma-aminobutyraldehyde dehydrogenase. J Biol Chem 234(8): 2145-2150

Jin Z, Mendu SK, Birnir B (2013) GABA is an effective immunomodulatory molecule. Amino Acids 45(1):87-94. doi:10.1007/s00726011-1193-7

Johnson JD, Mehus JG, Tews K, Milavetz BI, Lambeth DO (1998a) Genetic evidence for the expression of ATP- and GTP-specific succinyl-CoA synthetases in multicellular eucaryotes. J Biol Chem 273(42):27580-27586

Johnson JD, Muhonen WW, Lambeth DO (1998b) Characterization of the ATP- and GTP-specific succinyl-CoA synthetases in pigeon.
The enzymes incorporate the same alpha-subunit. J Biol Chem 273(42):27573-27579

Kadrmas EF, Ray PD, Lambeth DO (1991) Apparent ATP-linked succinate thiokinase activity and its relation to nucleoside diphosphate kinase in mitochondrial matrix preparations from rabbit. Biochim Biophys Acta 1074(3):339-346

Kiss G, Konrad C, Doczi J, Starkov AA, Kawamata H, Manfredi G et al (2013) The negative impact of alpha-ketoglutarate dehydrogenase complex deficiency on matrix substrate-level phosphorylation. FASEB J 27(6):2392-2406. doi:10.1096/fj.12-220202

Kiss G, Konrad C, Pour-Ghaz I, Mansour JJ, Nemeth B, Starkov AA et al (2014) Mitochondrial diaphorases as NAD $(+)$ donors to segments of the citric acid cycle that support substrate-level phosphorylation yielding ATP during respiratory inhibition. FASEB J 28(4):16821697. doi:10.1096/fj.13-243030

Kowluru A, Tannous M, Chen HQ (2002) Localization and characterization of the mitochondrial isoform of the nucleoside diphosphate kinase in the pancreatic beta cell: evidence for its complexation with mitochondrial succinyl-CoA synthetase. Arch Biochem Biophys 398(2):160-169

Labbe RF, Kurumada T, Onisawa J (1965) The role of succinyl-CoA synthetase in the control of heme biosynthesis. Biochim Biophys Acta 111(2):403-415

Lambeth DO, Tews KN, Adkins S, Frohlich D, Milavetz BI (2004) Expression of two succinyl-CoA synthetases with different nucleotide specificities in mammalian tissues. J Biol Chem 279(35):36621-36624

Lee M, Schwab C, McGeer PL (2011a) Astrocytes are GABAergic cells that modulate microglial activity. Glia 59(1):152-165. doi:10.1002/ glia. 21087

Lee M, McGeer EG, McGeer PL (2011b) Mechanisms of GABA release from human astrocytes. Glia 59(11):1600-1611. doi:10.1002/glia. 21202

Li X, Wu F, Beard DA (2013) Identification of the kinetic mechanism of succinyl-CoA synthetase. Biosci Rep 33(1):145-163. doi:10.1042/ BSR20120069

Lopes-Cardozo M, Larsson OM, Schousboe A (1986) Acetoacetate and glucose as lipid precursors and energy substrates in primary cultures of astrocytes and neurons from mouse cerebral cortex. J Neurochem 46(3):773-778

McKenna MC, Sonnewald U (2005) GABA alters the metabolic fate of [U-13C]glutamate in cultured cortical astrocytes. J Neurosci Res 79(1-2):81-87. doi:10.1002/jnr.20309

Mills E, O'Neill LA (2014) Succinate: a metabolic signal in inflammation. Trends Cell Biol 24(5):313-320. doi:10.1016/j.tcb.2013.11. 008

Minelli A, DeBiasi S, Brecha NC, Zuccarello LV, Conti F (1996) GAT-3, a high-affinity GABA plasma membrane transporter, is localized to astrocytic processes, and it is not confined to the vicinity of GABAergic synapses in the cerebral cortex. J Neurosci 16(19): 6255-6264

Murin R, Mohammadi G, Leibfritz D, Hamprecht B (2009a) Glial metabolism of valine. Neurochem Res 34(7):1195-1203. doi:10.1007/ s11064-008-9895-2

Murin R, Mohammadi G, Leibfritz D, Hamprecht B (2009b) Glial metabolism of isoleucine. Neurochem Res 34(1):194-204. doi:10. 1007/s11064-008-9840-4

Ottaway JH, McClellan JA, Saunderson CL (1981) Succinic thiokinase and metabolic control. Int J Biochem 13(4):401-410

Pall ML (1985) GTP: a central regulator of cellular anabolism. Curr Top Cell Regul 25:1-20

Pfaff E, Klingenberg M, Heldt HW (1965) Unspecific permeation and specific exchange of adenine nucleotides in liver mitochondria. Biochim Biophys Acta 104(1):312-315

Picklo MJ Sr, Olson SJ, Hayes JD, Markesbery WR, Montine TJ (2001) Elevation of AKR7A2 (succinic semialdehyde reductase) in neurodegenerative disease. Brain Res 916(1-2):229-238 
Plaitakis A, Zaganas I (2001) Regulation of human glutamate dehydrogenases: implications for glutamate, ammonia and energy metabolism in brain. J Neurosci Res 66(5):899-908

Ramirez A, van der Flier WM, Herold C, Ramonet D, Heilmann S, Lewczuk P et al (2014) SUCLG2 identified as both a determinator of CSF Abeta1-42 levels and an attenuator of cognitive decline in Alzheimer's disease. Hum Mol Genet. doi:10.1093/hmg/ddu372

Ris MM, von Wartburg JP (1973) Heterogeneity of NADPH-dependent aldehyde reductase from human and rat brain. Eur J Biochem 37(1): 69-77

Robinson AM, Williamson DH (1980) Physiological roles of ketone bodies as substrates and signals in mammalian tissues. Physiol Rev 60(1):143-187

Schousboe A, Westergaard N, Waagepetersen HS, Larsson OM, Bakken IJ, Sonnewald U (1997) Trafficking between glia and neurons of TCA cycle intermediates and related metabolites. Glia 21(1):99-105

Schwab C, Yu S, Wong W, McGeer EG, McGeer PL (2013) GAD65, GAD67, and GABAT immunostaining in human brain and apparent GAD65 loss in Alzheimer's disease. J Alzheimers Dis 33(4):10731088. doi:10.3233/JAD-2012-121330

Seiler N, al-Therib MJ, Kataoka K (1973) Formation of GABA from putrescine in the brain of fish (Salmo irideus Gibb.). J Neurochem 20(3):699-708

Sequerra EB, Gardino P, Hedin-Pereira C, de Mello FG (2007) Putrescine as an important source of GABA in the postnatal rat subventricular zone. Neuroscience 146(2):489-493. doi:10.1016/j.neuroscience. 2007.01.062
Soghomonian JJ, Martin DL (1998) Two isoforms of glutamate decarboxylase: why? Trends Pharmacol Sci 19(12):500-505

Spanaki C, Zaganas I, Kleopa KA, Plaitakis A (2010) Human GLUD2 glutamate dehydrogenase is expressed in neural and testicular supporting cells. J Biol Chem 285(22):16748-16756. doi:10.1074/ jbc.M109.092999

Spanaki C, Zaganas I, Kounoupa Z, Plaitakis A (2012) The complex regulation of human glud1 and glud2 glutamate dehydrogenases and its implications in nerve tissue biology. Neurochem Int 61(4):470 481. doi:10.1016/j.neuint.2012.05.020

Spanaki C, Kotzamani D, Petraki Z, Drakos E, Plaitakis A (2014) Heterogeneous cellular distribution of glutamate dehydrogenase in brain and in non-neural tissues. Neurochem Res 39(3):500-515. doi: 10.1007/s11064-013-1235-5

Velez-Fort M, Audinat E, Angulo MC (2012) Central role of GABA in neuron-glia interactions. Neuroscientist 18(3):237-250. doi:10. $1177 / 1073858411403317$

Westergaard N, Sonnewald U, Schousboe A (1994) Release of alphaketoglutarate, malate and succinate from cultured astrocytes: possible role in amino acid neurotransmitter homeostasis. Neurosci Lett 176(1):105-109

Zaganas I, Spanaki C, Plaitakis A (2012) Expression of human GLUD2 glutamate dehydrogenase in human tissues: functional implications. Neurochem Int 61(4):455-462. doi:10.1016/j.neuint.2012.06.007

Zhang Z, Tan M, Xie Z, Dai L, Chen Y, Zhao Y (2011) Identification of lysine succinylation as a new post-translational modification. Nat Chem Biol 7(1):58-63. doi:10.1038/nchembio.495 